KYUNGPOOK Math. J. 53(2013), 419-433

http://dx.doi.org/10.5666/KMJ.2013.53.3.419

\title{
A General System of Nonlinear Functional Equations in Non- Archimedean Spaces
}

\author{
Mohammad Bagher Ghaemi and Hamid Majani* \\ Department of Mathematics, Iran University of Science and Technology, Narmak, \\ Tehran, Iran \\ e-mail: mghaemi@iust.ac.ir and majani.hamid@hotmail.com \\ MADJID Eshaghi GordJI \\ Department of Mathematics, Semnan University, P. O. Box 35195-363, Semnan, \\ Iran \\ e-mail : madjid.eshaghi@gmail.com
}

Abstract. In this paper, we prove the generalized Hyers-Ulam-Rassias stability for a system of functional equations, called general system of nonlinear functional equations, in non-Archimedean normed spaces and Menger probabilistic non-Archimedean normed spaces.

\section{Introduction and Preliminaries}

The first stability problem concerning group homomorphisms was raised by Ulam[31] in 1940 and solved in the next year by Hyers[15]. Hyers' theorem was generalized by Aoki[2] for additive mappings and by Rassias[28] for linear mappings by considering an unbounded Cauchy difference. In 1994, a generalization of the Rassias theorem was obtained by Găvruta[11] by replacing the unbounded Cauchy difference by a general control function. In recent years many authors have investigated the stability of various functional equations in various spaces (see for instance $[4,6,7,8,12,16,17,21,26,27])$.

In this paper we establish some stability result concerning a general system of nonlinear functional equations in non-Archimedean normed spaces and Menger probabilistic non-Archimedean normed spaces.

It has been turned out that non-Archimedean spaces have many useful applications in quantum physics, p-adic strings and superstrings (see $[5,18,19,24])$. The proofs for non-Archimedean spaces are essentially different and entirely require new kind of intuition (see for instance $[3,9,10,23,25,32]$ ).

* Corresponding Author.

Received July 14, 2011; accepted January 18, 2013.

2010 Mathematics Subject Classification: 39B82, 39B52, 46S50.

Key words and phrases: Nonlinear Functional Equations, non-Archimedean Normed spaces, Generalized Hyers-Ulam-Rassias stability. 
Definition 1.1. Let $K$ be a field. A valuation mapping on $K$ is a function $|\cdot|: K \rightarrow R$ such that for any $a, b \in K$ we have

(i) $|a| \geq 0$ and equality holds if and only if $a=0$,

(ii) $|a b|=|a||b|$,

(iii) $|a+b| \leq|a|+|b|$.

A field endowed with a valuation mapping will be called a valued field. If the condition (iii) in the definition of a valuation mapping is replaced with

$$
\text { (iii) })^{\prime}|a+b| \leq \max \{|a|,|b|\}
$$

then the valuation $|\cdot|$ is said to be non-Archimedean. The condition(iii)' is called the strict triangle inequality. By (ii), we have $|1|=|-1|=1$. Thus, by induction, it follows from (iii) that $|n| \leq 1$ for each integer $n$. We always assume in addition that $|\cdot|$ is non trivial, i.e., that there is an $a_{0} \in K$ such that $\left|a_{0}\right| \notin\{0,1\}$. The most important examples of non-Archimedean spaces are p-adic numbers.

Example 1.2. Let $p$ be a prime number. For any non-zero rational number $a=p^{r} \frac{m}{n}$ such that $m$ and $n$ are coprime to the prime number $p$, define the $\mathrm{p}$-adic absolute value $|a|_{p}=p^{-r}$. Then $|\cdot|$ is a non-Archimedean norm on $\mathbb{Q}$. The completion of $\mathbb{Q}$ with respect to $|\cdot|$ is denoted by $\mathbb{Q}_{p}$ and is called the p-adic number field.

Definition 1.3. Let $X$ be a linear space over a scalar field $K$ with a nonArchimedean non-trivial valuation $|\cdot|$. A function $\|\cdot\|: X \rightarrow R$ is a nonArchimedean norm (valuation) if it satisfies the following conditions:

(NA1) $\|x\|=0$ if and only if $x=0$;

(NA2) $\|r x\|=|r|\|x\|$ for all $r \in K$ and $x \in X$;

(NA3) the strong triangle inequality (ultrametric); namely,

$$
\|x+y\| \leq \max \{\|x\|,\|y\|\} \quad(x, y \in X) .
$$

Then $(X,\|\cdot\|)$ is called a non-Archimedean space.

It follows from $(N A 3)$ that

$$
\left\|x_{m}-x_{l}\right\| \leq \max \left\{\left\|x_{\jmath+1}-x_{\jmath}\right\|: l \leq \jmath \leq m-1\right\} \quad(m>l),
$$

therefore a sequence $\left\{x_{m}\right\}$ is Cauchy in $X$ if and only if $\left\{x_{m+1}-x_{m}\right\}$ converges to zero in a non-Archimedean space.

Probabilistic normed spaces were first defined by Šerstnev in 1962 (see [30]). Their definition was generalized by Alsina, Schewizer and Sklar [1]. We recall and apply the definition of Menger probabilistic normed spaces briefly as given in [29]. 
Definition 1.4. A distance distribution function (briefly, a d.d.f.) is a nondecreasing function $F$ from $[0,+\infty]$ into $[0,1]$ that satisfies $F(0)=0$ and $F(+\infty)=$ 1 , and is left-continuous on $(0,+\infty)$. The space of d.d.f.'s will be denoted by $\Delta^{+}$; and the set of all $F$ in $\Delta^{+}$for which $\lim _{t \rightarrow+\infty^{-}} F(t)=1$ by $D^{+}$. The space $\Delta^{+}$ is partially ordered by the usual pointwise ordering of functions, i.e., $F \leq G$ if and only if $F(x) \leq G(x)$ for all $\mathrm{x}$ in $[0,+\infty]$. For any $a \geq 0, \varepsilon_{a}$ is the d.d.f. given by

$$
\varepsilon_{a}(t)= \begin{cases}0, & \text { if } t \leq a, \\ 1, & \text { if } t>a .\end{cases}
$$

Definition 1.5. A triangular norm (briefly $t$-norm) is a binary operation $T$ : $[0,1] \times[0,1] \rightarrow[0,1]$ which is commutative, associative, non-decreasing in each variable and has 1 as the unit element. Basic examples are the Eukasiewicz $t$-norm $T_{L}, T_{L}(a, b)=\max (a+b-1,0)$, the product $t$-norm $T_{P}, T_{P}(a, b)=a b$ and the strongest triangular norm $T_{M}, T_{M}(a, b)=\min (a, b)$.

Definition 1.6. A Menger Probabilistic Normed space is a triple $(X, \nu, T)$, where $X$ is a real vector space, $\mathrm{T}$ is continuous t-norm and $\nu$ is a mapping (the probabilistic norm) from $X$ into $\Delta^{+}$, such that for every choice of $p$ and $q$ in $X$ and $a, s, t$ in $(0,+\infty)$, the following hold:

(PN1) $\nu(p)=\varepsilon_{0}$, if and only if, $p=\theta \quad(\theta$ is the null vector in $X)$;

$(\mathrm{PN} 2) \nu(a p)(t)=\nu(p)\left(\frac{t}{|a|}\right)$

$(\mathrm{PN} 3) \nu(p+q)(s+t) \geq T(\nu(p)(s), \nu(q)(t))$.

Now we introduce definition of a Menger probabilistic non-Archimedean normed space by the definition of a non-Archimedean fuzzy normed space which is given in [20] and [22].

Definition 1.7. Let $X$ be a vector space over a non-Archimedean field $K$ and $T$ be a continuous $t$-norm. A triple $(X, \nu, T)$ is said to be a Menger probabilistic non-Archimedean normed space if (PN1) and (PN2) (in Definition??) and

$$
(P N A 3) \nu(x+y)(\max \{s, t\}) \geq T(\nu(x)(s), \nu(y)(t)),
$$

for all $x, y \in X$ and all $s, t>0$, are satisfied.

It follows from $\nu(x) \in \Delta^{+}$that $\nu(x)$ is non-decreasing for every $x \in X$. So one can show that the condition ( $P N A 3)$ is equivalent to the following condition:

$$
\nu(x+y)(t) \geq T(\nu(x)(t), \nu(y)(t)) .
$$


Definition 1.8. Let $(X, \nu, T)$ be a Menger probabilistic non-Archimedean normed space. Let $\left\{x_{n}\right\}$ be a sequence in $X$. Then $\left\{x_{n}\right\}$ is said to be convergent if there exists $x \in X$ such that $\lim _{n \rightarrow \infty} \nu\left(x_{n}-x\right)(t)=1$, for all $t>0$. In that case, $x$ is called the limit of the sequence $\left\{x_{n}\right\}$. A sequence $\left\{x_{n}\right\}$ in $\mathrm{X}$ is called Cauchy if for each $\varepsilon>0$ and each $t>0$ there exists $n_{0}$ such that for all $n \geq n_{0}$ and all $p>0$ we have $\nu\left(x_{n+p}-x_{n}\right)(t)>1-\varepsilon$.

Let $T$ be a given t-norm. Then (by associativity) a family of mappings $T^{n}$ : $[0,1] \rightarrow[0,1], n \in \mathbb{N}$, is defined as follows:

$$
T^{1}(x)=T(x, x), T^{n}(x)=T\left(T^{n-1}(x), x\right), x \in[0,1] .
$$

For three important t-norms $T_{M}, T_{P}$ and $T_{L}$ we have

$$
T_{M}^{n}(x)=x, T_{P}^{n}(x)=x^{n}, T_{L}^{n}(x)=\max \{(n+1) x-n, 0\}, n \in \mathbb{N} .
$$

Definition 1.9(Hadzićc[13]). A t-norm $T$ is said to be of $\mathrm{H}$-type if a family of functions $\left\{T^{n}(t)\right\} ; n \in \mathbb{N}$, is equicontinuous at $t=1$, that is,

$$
\forall \varepsilon \in(0,1) \exists \delta \in(0,1): t>1-\delta \Rightarrow T^{n}(t)>1-\varepsilon(n \geq 1) .
$$

The t-norm $T_{M}$ is a trivial example of $\mathrm{t}$-norm of $\mathrm{H}$-type, but there are t-norms of H-type with $T \neq T_{M}$ (see e.g., Hadzić[14]).

Lemma 1.10. We consider the notations of the Definition(1.8.). Also assume that $T$ is a $t$-norm of H-type. Then the sequence $\left\{x_{n}\right\}$ is Cauchy if for each $\varepsilon>0$ and each $t>0$ there exists $n_{0}$ such that for all $n \geq n_{0}$ we have $\nu\left(x_{n+1}-x_{n}\right)(t)>1-\varepsilon$.

Proof. Due to

$$
\begin{aligned}
& \nu\left(x_{n+p}-x_{n}\right)(t) \geq T\left(\nu\left(x_{n+p}-x_{n+p-1}\right)(t), \nu\left(x_{n+p-1}-x_{n}\right)(t)\right) \geq \\
& T\left(\nu\left(x_{n+p}-x_{n+p-1}\right)(t), T\left(\nu\left(x_{n+p-1}-x_{n+p-2}\right)(t), \nu\left(x_{n+p-2}-x_{n}\right)(t)\right)\right) \geq \\
& \quad \quad \quad \\
& \geq T\left(\nu\left(x_{n+p}-x_{n+p-1}\right)(t), T\left(\nu\left(x_{n+p-1}-x_{n+p-2}\right)(t), \cdots,\right.\right. \\
& \left.\left.T\left(\nu\left(x_{n+2}-x_{n+1}\right)(t), \nu\left(x_{n+1}-x_{n}\right)(t)\right)\right) \cdots\right),
\end{aligned}
$$

and by the assumption of $\mathrm{T}$, which is an H-type t-norm, the sequence $\left\{x_{n}\right\}$ is Cauchy if for each $\varepsilon>0$ and each $t>0$ there exists $n_{0}$ such that for all $n \geq n_{0}$ we have $\nu\left(x_{n+1}-x_{n}\right)(t)>1-\varepsilon$. We will use this criterion in this paper.

It is easy to see that every convergent sequence in a (Menger probabilistic) nonArchimedean normed space is Cauchy. If each Cauchy sequence is convergent, then 
the (Menger probabilistic) non-Archimedean normed space is said to be complete and is called (Menger probabilistic) non-Archimedean Banach space.

We assume that $f: X^{n} \rightarrow Y$ and $\lambda_{i}: \mathbb{K} \rightarrow \mathbb{K}$ be mappings and introduce the following system:

$$
\left\{\begin{array}{l}
f\left(a_{1} x_{1}, x_{2}, \ldots, x_{n}\right)=\lambda_{1}\left(a_{1}\right) f\left(x_{1}, \ldots, x_{n}\right) ; \\
f\left(x_{1}, a_{2} x_{2}, \ldots, x_{n}\right)=\lambda_{2}\left(a_{2}\right) f\left(x_{1}, \ldots, x_{n}\right) ; \\
\quad \vdots \\
f\left(x_{1}, \ldots, a_{i} x_{i}, \ldots, x_{n}\right)=\lambda_{i}\left(a_{i}\right) f\left(x_{1}, \ldots, x_{n}\right) ; \\
\quad \vdots \\
f\left(x_{1}, \ldots, x_{n-1}, a_{n} x_{n}\right)=\lambda_{n}\left(a_{n}\right) f\left(x_{1}, \ldots, x_{n}\right) ;
\end{array}\right.
$$

for all $x_{i} \in X$ and $a_{i} \in \mathbb{K} \backslash\{0\}, i=1, \ldots, n$. We call the above system the general system of nonlinear functional equations. One can show that the following mappings satisfying system(1.1).

$$
\left\{\begin{array}{l}
\lambda_{i}(a)=a^{i} \Longrightarrow f\left(x_{1}, x_{2}, \ldots, x_{n}\right)=x_{1} x_{2}^{2} \cdots x_{n}^{n} \\
\lambda_{i}(a)=b_{i}^{a^{i}} \text { for } b_{i} \neq 0, \pm 1 \Longrightarrow f\left(x_{1}, x_{2}, \ldots, x_{n}\right)=b_{1}^{x_{1}} b_{2}^{x_{2}^{2}} \cdots b_{n}^{x_{n}^{n}}
\end{array}\right.
$$

In the section(2), we establish the generalized Hyers-Ulam-Rassias stability of system(1.1) in non-Archimedean Banach spaces. In the section(3), we establish the generalized Hyers-Ulam-Rassias stability of system(1.1) in Menger probabilistic non-Archimedean Banach spaces.

\section{System(1.1) Stability in non-Archimedean Banach Spaces}

In this section, we prove the generalized Hyers-Ulam-Rassias stability of system(1.1) in non-Archimedean Banach spaces. Throughout this section, we assume that $i, m, n, p \in \mathbb{N} \cup\{0\}, K$ is a non-Archimedean field, $\mathrm{Y}$ is a nonArchimedean Banach space over $K$ and $X$ is a vector space over $K$. Also assume that $f: X^{n} \rightarrow Y$ and $\lambda_{i}: \mathbb{K} \rightarrow \mathbb{K}$ are mappings.

Theorem 2.1. Let $\varphi_{i}: X^{n} \rightarrow[0, \infty)$ for $i \in\{1, \ldots, n\}$ be a function such that

$$
\begin{aligned}
& \lim _{m \rightarrow \infty} \max \left\{\frac{1}{\left|\left(\lambda_{1}\left(a_{1}\right)\right)^{m+1} \ldots\left(\lambda_{i}\left(a_{i}\right)\right)^{m+1}\left(\lambda_{i+1}\left(a_{i+1}\right)\right)^{m} \ldots\left(\lambda_{n}\left(a_{n}\right)\right)^{m}\right|}\right. \\
& \left.\varphi_{i}\left(a_{1}^{m+1} x_{1}, \ldots, a_{i-1}^{m+1} x_{i-1}, a_{i}^{m} x_{i}, \ldots, a_{n}^{m} x_{n}\right): \quad i=1, \ldots, n\right\}=0,
\end{aligned}
$$


and

$$
\begin{aligned}
& \Phi=\Phi\left(x_{1}, \ldots, x_{n}\right)=\lim _{p \rightarrow \infty} \max \{ \\
& \max \left\{\frac{1}{\left|\left(\lambda_{1}\left(a_{1}\right)\right)^{m+1} \ldots\left(\lambda_{i}\left(a_{i}\right)\right)^{m+1}\left(\lambda_{i+1}\left(a_{i+1}\right)\right)^{m} \ldots\left(\lambda_{n}\left(a_{n}\right)\right)^{m}\right|}\right. \\
& \varphi_{i}\left(a_{1}^{m+1} x_{1}, \ldots, a_{i-1}^{m+1} x_{i-1}, a_{i}^{m} x_{i}, \ldots, a_{n}^{m} x_{n}\right) \\
& \quad: i=1, \ldots, n\}: m=0,1, \ldots, p\}<\infty,
\end{aligned}
$$

and

$$
\lim _{m \rightarrow \infty} \frac{1}{\left|\left(\lambda_{1}\left(a_{1}\right)\right)^{m} \ldots\left(\lambda_{n}\left(a_{n}\right)\right)^{m}\right|} \varphi_{i}\left(a_{1}^{m} x_{1}, \ldots, a_{n}^{m} x_{n}\right)=0,
$$

for all $x_{i} \in X$ and $a_{i} \in \mathbb{K} \backslash\{0\}, i=1, \ldots, n$. Let $f: X^{n} \rightarrow Y$ be a mapping satisfying

$$
\left\{\begin{array}{l}
\left\|f\left(a_{1} x_{1}, x_{2}, \ldots, x_{n}\right)-\lambda_{1}\left(a_{1}\right) f\left(x_{1}, \ldots, x_{n}\right)\right\| \leq \varphi_{1}\left(x_{1}, \ldots, x_{n}\right) \\
\quad \vdots \\
\left\|f\left(x_{1}, \ldots, a_{i} x_{i}, \ldots, x_{n}\right)-\lambda_{i}\left(a_{i}\right) f\left(x_{1}, \ldots, x_{n}\right)\right\| \leq \varphi_{i}\left(x_{1}, \ldots, x_{n}\right) \\
\quad \vdots \\
\left\|f\left(x_{1}, \ldots, x_{n-1}, a_{n} x_{n}\right)-\lambda_{n}\left(a_{n}\right) f\left(x_{1}, \ldots, x_{n}\right)\right\| \leq \varphi_{n}\left(x_{1}, \ldots, x_{n}\right)
\end{array}\right.
$$

for all $x_{i} \in X$ and $a_{i} \in \mathbb{K} \backslash\{0\}, i=1, \ldots, n$. Then there exists a unique mapping $T: X^{n} \rightarrow Y$ satisfying system(1.1) and

$$
\left\|f\left(x_{1}, \ldots, x_{n}\right)-T\left(x_{1}, \ldots, x_{n}\right)\right\| \leq \Phi,
$$

for all $x_{i} \in X, i=1, \ldots, n$.

Proof. Fix $i \in\{1,2, \ldots, n\}$ and consider the following inequality.

$$
\left\|f\left(x_{1}, \ldots, a_{i} x_{i}, \ldots, x_{n}\right)-\lambda_{i}\left(a_{i}\right) f\left(x_{1}, \ldots, x_{n}\right)\right\| \leq \varphi_{i}\left(x_{1}, \ldots, x_{n}\right) .
$$

From (2.5) we get

$$
\left\|f\left(x_{1}, \ldots, x_{n}\right)-\frac{1}{\lambda_{i}\left(a_{i}\right)} f\left(x_{1}, \ldots, a_{i} x_{i}, \ldots, x_{n}\right)\right\| \leq \frac{1}{\left|\lambda_{i}\left(a_{i}\right)\right|} \varphi_{i}\left(x_{1}, \ldots, x_{n}\right) .
$$

Therefore one can obtain

$$
\begin{aligned}
& \| \frac{1}{\lambda_{1}\left(a_{1}\right) \ldots \lambda_{i-1}\left(a_{i-1}\right)} f\left(a_{1} x_{1}, \ldots, a_{i-1} x_{i-1}, x_{i}, \ldots, x_{n}\right)- \\
& \frac{1}{\lambda_{1}\left(a_{1}\right) \ldots \lambda_{i}\left(a_{i}\right)} f\left(a_{1} x_{1}, \ldots, a_{i} x_{i}, x_{i+1}, \ldots, x_{n}\right) \| \leq \\
& \frac{1}{\left|\lambda_{1}\left(a_{1}\right) \ldots \lambda_{i}\left(a_{i}\right)\right|} \varphi_{i}\left(a_{1} x_{1}, \ldots, a_{i-1} x_{i-1}, x_{i}, \ldots, x_{n}\right) .
\end{aligned}
$$


So by induction and (2.6), we conclude

$$
\begin{aligned}
& \left\|f\left(x_{1}, \ldots, x_{n}\right)-\frac{1}{\lambda_{1}\left(a_{1}\right) \ldots \lambda_{n}\left(a_{n}\right)} f\left(a_{1} x_{1}, \ldots, a_{n} x_{n}\right)\right\| \leq \\
& \max \left\{\frac{1}{\left|\lambda_{1}\left(a_{1}\right) \ldots \lambda_{i}\left(a_{i}\right)\right|} \varphi_{i}\left(a_{1} x_{1}, \ldots, a_{i-1} x_{i-1}, x_{i}, \ldots, x_{n}\right): i=1, \ldots, n\right\} .
\end{aligned}
$$

Therefore we get

$$
\begin{aligned}
& \| \frac{1}{\left(\lambda_{1}\left(a_{1}\right)\right)^{m} \ldots\left(\lambda_{n}\left(a_{n}\right)\right)^{m}} f\left(a_{1}^{m} x_{1}, \ldots, a_{n}^{m} x_{n}\right)- \\
& \frac{1}{\left(\lambda_{1}\left(a_{1}\right)\right)^{m+1} \ldots\left(\lambda_{n}\left(a_{n}\right)\right)^{m+1}} f\left(a_{1}^{m+1} x_{1}, \ldots, a_{n}^{m+1} x_{n}\right) \| \leq \\
& \max \left\{\frac{1}{\left|\left(\lambda_{1}\left(a_{1}\right)\right)^{m+1} \ldots\left(\lambda_{i}\left(a_{i}\right)\right)^{m+1}\left(\lambda_{i+1}\left(a_{i+1}\right)\right)^{m} \ldots\left(\lambda_{n}\left(a_{n}\right)\right)^{m}\right|}\right. \\
& \left.\varphi_{i}\left(a_{1}^{m+1} x_{1}, \ldots, a_{i-1}^{m+1} x_{i-1}, a_{i}^{m} x_{i}, \ldots, a_{n}^{m} x_{n}\right): i=1, \ldots, n\right\},
\end{aligned}
$$

for all $m \in \mathbb{N} \cup\{0\}$. It follows from (2.7) and (2.1) that the sequence

$$
\left\{\frac{1}{\left(\lambda_{1}\left(a_{1}\right)\right)^{m} \ldots\left(\lambda_{n}\left(a_{n}\right)\right)^{m}} f\left(a_{1}^{m} x_{1}, \ldots, a_{n}^{m} x_{n}\right)\right\}
$$

is Cauchy. Since the space $Y$ is complete, this sequence is convergent. Therefore we can define

$T: X^{n} \rightarrow Y$ by

$$
T\left(x_{1}, \ldots, x_{n}\right):=\lim _{m \rightarrow \infty} \frac{1}{\left(\lambda_{1}\left(a_{1}\right)\right)^{m} \ldots\left(\lambda_{n}\left(a_{n}\right)\right)^{m}} f\left(a_{1}^{m} x_{1}, \ldots, a_{n}^{m} x_{n}\right)
$$

for all $x_{i} \in X$ and $a_{i} \in \mathbb{K} \backslash\{0\}, i=1, \ldots, n$. Using induction with (2.7) one can show that

$$
\begin{aligned}
& \left\|f\left(x_{1}, \ldots, x_{n}\right)-\frac{1}{\left(\lambda_{1}\left(a_{1}\right)\right)^{p} \ldots\left(\lambda_{n}\left(a_{n}\right)\right)^{p}} f\left(a_{1}^{p} x_{1}, \ldots, a_{n}^{p} x_{n}\right)\right\| \leq \\
& \max \left\{\operatorname { m a x } \left\{\frac{1}{\left|\left(\lambda_{1}\left(a_{1}\right)\right)^{m+1} \ldots\left(\lambda_{i}\left(a_{i}\right)\right)^{m+1}\left(\lambda_{i+1}\left(a_{i+1}\right)\right)^{m} \ldots\left(\lambda_{n}\left(a_{n}\right)\right)^{m}\right|}\right.\right. \\
& \varphi_{i}\left(a_{1}^{m+1} x_{1}, \ldots, a_{i-1}^{m+1} x_{i-1}, a_{i}^{m} x_{i}, \ldots, a_{n}^{m} x_{n}\right) \\
& : i=1, \ldots, n\}: m=0,1, \ldots, p\} .
\end{aligned}
$$

for all $x_{i} \in X, i=1, \ldots, n$ and $p \in \mathbb{N} \cup\{0\}$. By taking $p$ to approach infinity in (2.9) and using (2.2) one obtains (2.4). 
For fixed $i \in\{1,2, \ldots, n\}$ and by (2.5) and (2.8), we get

$$
\begin{aligned}
& \left\|T\left(x_{1}, \ldots, a_{i} x_{i}, \ldots, x_{n}\right)-\lambda_{i}\left(a_{i}\right) T\left(x_{1}, \ldots, x_{n}\right)\right\|= \\
& \lim _{m \rightarrow \infty} \| \frac{1}{\left(\lambda_{1}\left(a_{1}\right)\right)^{m} \ldots\left(\lambda_{n}\left(a_{n}\right)\right)^{m}} f\left(a_{1}^{m} x_{1}, \ldots, a_{i}^{m+1} x_{i}, \ldots, a_{n}^{m} x_{n}\right)- \\
& \frac{\lambda_{i}\left(a_{i}\right)}{\left(\lambda_{1}\left(a_{1}\right)\right)^{m} \ldots\left(\lambda_{n}\left(a_{n}\right)\right)^{m}} f\left(a_{1}^{m} x_{1}, \ldots, a_{n}^{m} x_{n}\right) \| \\
& \leq \lim _{m \rightarrow \infty} \frac{1}{\left|\left(\lambda_{1}\left(a_{1}\right)\right)^{m} \ldots\left(\lambda_{n}\left(a_{n}\right)\right)^{m}\right|} \varphi_{i}\left(a_{1}^{m} x_{1}, \ldots, a_{n}^{m} x_{n}\right) .
\end{aligned}
$$

By (2.10) and (2.3), we conclude that $T$ satisfies system(1.1).

Suppose that there exists another mapping $T^{\prime}: X^{n} \rightarrow Y$ which satisfies system(1.1) and (2.4). So we have

$$
\begin{aligned}
& \left\|T\left(x_{1}, x_{2}, \ldots, x_{n}\right)-T^{\prime}\left(x_{1}, x_{2}, \ldots, x_{n}\right)\right\| \leq \\
& \frac{1}{\left|\left(\lambda_{1}\left(a_{1}\right)\right)^{m} \ldots\left(\lambda_{n}\left(a_{n}\right)\right)^{m}\right|} \max \left\{\left\|T\left(a_{1}^{m} x_{1}, \ldots, a_{n}^{m} x_{n}\right)-f\left(a_{1}^{m} x_{1}, \ldots, a_{n}^{m} x_{n}\right)\right\|,\right. \\
& \left.\left\|f\left(a_{1}^{m} x_{1}, \ldots, a_{n}^{m} x_{n}\right)-T^{\prime}\left(a_{1}^{m} x_{1}, \ldots, a_{n}^{m} x_{n}\right)\right\|\right\} \leq \\
& \frac{1}{\left|\left(\lambda_{1}\left(a_{1}\right)\right)^{m} \ldots\left(\lambda_{n}\left(a_{n}\right)\right)^{m}\right|} \max \left\{\Phi\left(a_{1}^{m} x_{1}, \ldots, a_{n}^{m} x_{n}\right), \Phi\left(a_{1}^{m} x_{1}, \ldots, a_{n}^{m} x_{n}\right)\right\},
\end{aligned}
$$

which tends to zero as $m \rightarrow \infty$ by (2.2). Therefore $T=T^{\prime}$. This completes the proof.

We obtain the following corollary if we assume $\lambda_{i}(a)=a^{i}$ in Theorem(2.1).

Corollary 2.2. Let $\varphi_{i}: X^{n} \rightarrow[0, \infty)$ for $i \in\{1,2, \ldots, n\}$ be a function such that

$$
\begin{aligned}
& \qquad \lim _{m \rightarrow \infty} \max \left\{\frac { 1 } { | a _ { 1 } ^ { m + 1 } a _ { 2 } ^ { 2 ( m + 1 ) } \ldots a _ { i } ^ { i ( m + 1 ) } a _ { i + 1 } ^ { ( i + 1 ) m } \ldots a _ { n } ^ { n m } | } \varphi _ { i } \left(a_{1}^{m+1} x_{1}, \ldots, a_{i-1}^{m+1} x_{i-1},\right.\right. \\
& \left.\left.\qquad a_{i}^{m} x_{i}, \ldots, a_{n}^{m} x_{n}\right): i=1, \ldots, n\right\}=0, \\
& \text { and } \\
& \Phi=\Phi\left(x_{1}, \ldots, x_{n}\right)= \\
& \lim _{p \rightarrow \infty} \max \left\{\operatorname { m a x } \left\{\frac { 1 } { | a _ { 1 } ^ { m + 1 } a _ { 2 } ^ { 2 ( m + 1 ) } \ldots a _ { i } ^ { i ( m + 1 ) } a _ { i + 1 } ^ { ( i + 1 ) m } \ldots a _ { n } ^ { n m } | } \varphi _ { i } \left(a_{1}^{m+1} x_{1}, \ldots, a_{i-1}^{m+1} x_{i-1},\right.\right.\right. \\
& \left.\left.\left.a_{i}^{m} x_{i}, \ldots, a_{n}^{m} x_{n}\right): i=1, \ldots, n\right\}: m=0,1, \ldots, p\right\}<\infty,
\end{aligned}
$$

and

$$
\lim _{m \rightarrow \infty} \frac{1}{\left|a_{1}^{m} a_{2}^{2 m} \ldots a_{n}^{n m}\right|} \varphi_{i}\left(a_{1}^{m} x_{1}, \ldots, a_{n}^{m} x_{n}\right)=0
$$


for all $x_{i} \in X$ and $a_{i} \in \mathbb{K} \backslash\{0\}, i=1, \ldots, n$. Let $f: X^{n} \rightarrow Y$ be a mapping satisfying

$$
\left\{\begin{array}{l}
\left\|f\left(a_{1} x_{1}, x_{2}, \ldots, x_{n}\right)-a_{1} f\left(x_{1}, \ldots, x_{n}\right)\right\| \leq \varphi_{1}\left(x_{1}, \ldots, x_{n}\right) \\
\left\|f\left(x_{1}, a_{2} x_{2}, \ldots, x_{n}\right)-a_{2}^{2} f\left(x_{1}, \ldots, x_{n}\right)\right\| \leq \varphi_{2}\left(x_{1}, \ldots, x_{n}\right) ; \\
\quad \vdots \\
\left\|f\left(x_{1}, \ldots, a_{i} x_{i}, \ldots, x_{n}\right)-a_{i}^{i} f\left(x_{1}, \ldots, x_{n}\right)\right\| \leq \varphi_{i}\left(x_{1}, \ldots, x_{n}\right) ; \\
\quad \vdots \\
\left\|f\left(x_{1}, \ldots, x_{n-1}, a_{n} x_{n}\right)-a_{n}^{n} f\left(x_{1}, \ldots, x_{n}\right)\right\| \leq \varphi_{n}\left(x_{1}, \ldots, x_{n}\right) ;
\end{array}\right.
$$

for all $x_{i} \in X$ and $a_{i} \in \mathbb{K} \backslash\{0\}, i=1, \ldots, n$. Then there exists a unique mapping $T: X^{n} \rightarrow Y$ satisfying system(1.1) for $\lambda_{i}\left(a_{i}\right)=a_{i}^{i}$ and

$$
\left\|f\left(x_{1}, \ldots, x_{n}\right)-T\left(x_{1}, \ldots, x_{n}\right)\right\| \leq \Phi,
$$

for all $x_{i} \in X, i=1, \ldots, n$.

\section{System (1.1) Stability in Menger Probabilistic non-Archimedean Ba- nach Spaces}

In this section, we prove the generalized Hyers-Ulam-Rassias stability of system(1.1) in Menger probabilistic non-Archimedean Banach spaces. Throughout this section, we assume that $u \in \mathbb{R}, i, m, n \in \mathbb{N} \cup\{0\}, K$ is a non-Archimedean field, $T$ is a continuous $\mathrm{t}$-norm of $\mathrm{H}$-type, $(Y, \nu, T)$ is a Menger probabilistic non-Archimedean Banach space over $K,(Z, \omega, T)$ is a Menger probabilistic nonArchimedean normed space over $K$ and $X$ is a vector space over $K$. Also assume that $f: X^{n} \rightarrow Y$ and $\lambda_{i}: \mathbb{K} \rightarrow \mathbb{K}$ are mappings.

Theorem 3.1. Let $\varphi_{i}: X^{n} \rightarrow Z$ for $i \in\{1, \ldots, n\}$ be a mapping such that

$$
\left\{\begin{array}{l}
\tilde{\varphi}_{i}=\tilde{\varphi}_{i}\left(x_{1}, \ldots, x_{n}, u\right)= \\
\omega\left(\frac{1}{\left|\lambda_{1}\left(a_{1}\right) \ldots \lambda_{i}\left(a_{i}\right)\right|} \varphi_{i}\left(a_{1} x_{1}, \ldots, a_{i-1} x_{i-1}, x_{i}, \ldots, x_{n}\right)\right)(u) \\
\Phi_{1}=\Phi_{1}\left(x_{1}, \ldots, x_{n}, u\right)=\tilde{\varphi}_{1}\left(x_{1}, \ldots, x_{n}, u\right) \\
\Phi_{i}=\Phi_{i}\left(x_{1}, \ldots, x_{n}, u\right)=T\left(\tilde{\varphi}_{i}\left(x_{1}, \ldots, x_{n}, u\right), \Phi_{i-1}\left(x_{1}, \ldots, x_{n}, u\right)\right) \\
\lim _{m \rightarrow \infty} \Phi_{n}\left(a_{1}^{m} x_{1}, \ldots, a_{n}^{m} x_{n},\left|\left(\lambda_{1}\left(a_{1}\right)\right)^{m} \ldots\left(\lambda_{n}\left(a_{n}\right)\right)^{m}\right| u\right)=1
\end{array}\right.
$$

and

$$
\lim _{m \rightarrow \infty} \omega\left(\frac{1}{\left|\left(\lambda_{1}\left(a_{1}\right)\right)^{m} \ldots\left(\lambda_{n}\left(a_{n}\right)\right)^{m}\right|} \varphi_{i}\left(a_{1}^{m} x_{1}, \ldots, a_{n}^{m} x_{n}\right)\right)(u)=1
$$


and

$$
\left\{\begin{array}{l}
\Phi_{m}^{*}=\Phi_{m}^{*}\left(x_{1}, \ldots, x_{n}, u\right)= \\
\Phi_{n}\left(a_{1}^{m} x_{1}, \ldots, a_{n}^{m} x_{n},\left|\left(\lambda_{1}\left(a_{1}\right)\right)^{m} \ldots\left(\lambda_{n}\left(a_{n}\right)\right)^{m}\right| u\right) \\
\Psi_{0}=\Phi_{0}^{*}\left(x_{1}, \ldots, x_{n}, u\right)=\Phi_{n}\left(x_{1}, \ldots, x_{n}, u\right) \\
\Psi_{m}=\Psi_{m}\left(x_{1}, \ldots, x_{n}, u\right)= \\
T\left(\Phi_{m}^{*}\left(x_{1}, \ldots, x_{n}, u\right), \Psi_{m-1}\left(x_{1}, \ldots, x_{n}, u\right)\right) \\
\Psi=\Psi\left(x_{1}, \ldots, x_{n}, u\right)=\lim _{m \rightarrow \infty} \Psi_{m}=1
\end{array}\right.
$$

for all $u>0, x_{i} \in X$ and $a_{i} \in \mathbb{K} \backslash\{0\}, i=1, \ldots, n$. Let $f: X^{n} \rightarrow Y$ be a mapping satisfying

$$
\left\{\begin{array}{l}
\nu\left(f\left(a_{1} x_{1}, x_{2}, \ldots, x_{n}\right)-\lambda_{1}\left(a_{1}\right) f\left(x_{1}, \ldots, x_{n}\right)\right)(u) \geq \omega\left(\varphi_{1}\left(x_{1}, \ldots, x_{n}\right)\right)(u) ; \\
\quad \vdots \\
\nu\left(f\left(x_{1}, \ldots, a_{i} x_{i}, \ldots, x_{n}\right)-\lambda_{i}\left(a_{i}\right) f\left(x_{1}, \ldots, x_{n}\right)\right)(u) \geq \omega\left(\varphi_{i}\left(x_{1}, \ldots, x_{n}\right)\right)(u) ; \\
\quad \vdots \\
\nu\left(f\left(x_{1}, \ldots, x_{n-1}, a_{n} x_{n}\right)-\lambda_{n}\left(a_{n}\right) f\left(x_{1}, \ldots, x_{n}\right)\right)(u) \geq \omega\left(\varphi_{n}\left(x_{1}, \ldots, x_{n}\right)\right)(u) ;
\end{array}\right.
$$

for all $u>0, x_{i} \in X$ and $a_{i} \in \mathbb{K} \backslash\{0\}, i=1, \ldots, n$. Then there exists a unique mapping $F: X^{n} \rightarrow Y$ satisfying system(1.1) and

$$
\nu\left(f\left(x_{1}, \ldots, x_{n}\right)-F\left(x_{1}, \ldots, x_{n}\right)\right)(u) \geq \Psi,
$$

for all $u>0$ and $x_{i} \in X, i=1, \ldots, n$.

Proof. Fix $i \in\{1,2, \ldots, n\}$ and consider the following inequality.

$$
\begin{aligned}
& \nu\left(f\left(x_{1}, \ldots, a_{i} x_{i}, \ldots, x_{n}\right)-\lambda_{i}\left(a_{i}\right) f\left(x_{1}, \ldots, x_{n}\right)\right)(u) \geq \\
& \omega\left(\varphi_{i}\left(x_{1}, \ldots, x_{n}\right)\right)(u) .
\end{aligned}
$$

From (3.5) we get

$$
\nu\left(f\left(x_{1}, \ldots, x_{n}\right)-\frac{1}{\lambda_{i}\left(a_{i}\right)} f\left(x_{1}, \ldots, a_{i} x_{i}, \ldots, x_{n}\right)\right)(u) \geq \omega\left(\frac{1}{\left|\lambda_{i}\left(a_{i}\right)\right|} \varphi_{i}\left(x_{1}, \ldots, x_{n}\right)\right)(u) .
$$

Therefore one can obtain

$$
\begin{aligned}
& \nu\left(\frac{1}{\lambda_{1}\left(a_{1}\right) \ldots \lambda_{i-1}\left(a_{i-1}\right)} f\left(a_{1} x_{1}, \ldots, a_{i-1} x_{i-1}, x_{i}, \ldots, x_{n}\right)-\right. \\
& \left.\frac{1}{\lambda_{1}\left(a_{1}\right) \ldots \lambda_{i}\left(a_{i}\right)} f\left(a_{1} x_{1}, \ldots, a_{i} x_{i}, x_{i+1}, \ldots, x_{n}\right)\right)(u) \geq \\
& \omega\left(\frac{1}{\left|\lambda_{1}\left(a_{1}\right) \ldots \lambda_{i}\left(a_{i}\right)\right|} \varphi_{i}\left(a_{1} x_{1}, \ldots, a_{i-1} x_{i-1}, x_{i}, \ldots, x_{n}\right)\right)(u)=\tilde{\varphi}_{i} .
\end{aligned}
$$


So by induction and by (3.1) and (3.6), we have

$$
\nu\left(f\left(x_{1}, \ldots, x_{n}\right)-\frac{1}{\lambda_{1}\left(a_{1}\right) \ldots \lambda_{n}\left(a_{n}\right)} f\left(a_{1} x_{1}, \ldots, a_{n} x_{n}\right)\right)(u) \geq \Phi_{n} .
$$

Therefore we get

$$
\begin{aligned}
& \nu\left(\frac{1}{\left(\lambda_{1}\left(a_{1}\right)\right)^{m} \ldots\left(\lambda_{n}\left(a_{n}\right)\right)^{m}} f\left(a_{1}^{m} x_{1}, \ldots, a_{n}^{m} x_{n}\right)-\right. \\
& \left.\frac{1}{\left(\lambda_{1}\left(a_{1}\right)\right)^{m+1} \ldots\left(\lambda_{n}\left(a_{n}\right)\right)^{m+1}} f\left(a_{1}^{m+1} x_{1}, \ldots, a_{n}^{m+1} x_{n}\right)\right)(u) \geq \\
& \Phi_{n}\left(a_{1}^{m} x_{1}, \ldots, a_{n}^{m} x_{n},\left|\left(\lambda_{1}\left(a_{1}\right)\right)^{m} \ldots\left(\lambda_{n}\left(a_{n}\right)\right)^{m}\right| u\right)
\end{aligned}
$$

for all $m \in \mathbb{N} \cup\{0\}$. So by (3.1) and (3.8), the sequence

$$
\left\{\frac{1}{\left(\lambda_{1}\left(a_{1}\right)\right)^{m} \ldots\left(\lambda_{n}\left(a_{n}\right)\right)^{m}} f\left(a_{1}^{m} x_{1}, \ldots, a_{n}^{m} x_{n}\right)\right\}
$$

is Cauchy. By completeness of $Y$, we conclude that it is convergent. Therefore we can define $F: X^{n} \rightarrow Y$ by

$$
\begin{aligned}
& \lim _{m \rightarrow \infty} \nu\left(F\left(x_{1}, \ldots, x_{n}\right)-\right. \\
& \left.\frac{1}{\left(\lambda_{1}\left(a_{1}\right)\right)^{m} \ldots\left(\lambda_{n}\left(a_{n}\right)\right)^{m}} f\left(a_{1}^{m} x_{1}, \ldots, a_{n}^{m} x_{n}\right)\right)(u)=1,
\end{aligned}
$$

for all $u>0, x_{i} \in X$ and $a_{i} \in \mathbb{K} \backslash\{0\}, i=1, \ldots, n$. Using induction with (3.8) one can show that

$$
\begin{aligned}
& \nu\left(f\left(x_{1}, \ldots, x_{n}\right)-\right. \\
& \left.\frac{1}{\left(\lambda_{1}\left(a_{1}\right)\right)^{m} \ldots\left(\lambda_{n}\left(a_{n}\right)\right)^{m}} f\left(a_{1}^{m} x_{1}, \ldots, a_{n}^{m} x_{n}\right)\right)(u) \geq \Psi_{m} .
\end{aligned}
$$

By taking $m$ to approach infinity in (3.10) and using (3.3) one obtains (3.4).

For $i \in\{1,2, \ldots, n\}$ and by (3.5) and (3.9), we get

$$
\begin{aligned}
& \nu\left(F\left(x_{1}, \ldots, a_{i} x_{i}, \ldots, x_{n}\right)-\lambda_{i}\left(a_{i}\right) F\left(x_{1}, \ldots, x_{n}\right)\right)(u)= \\
& \lim _{m \rightarrow \infty} \nu\left(\frac{1}{\left(\lambda_{1}\left(a_{1}\right)\right)^{m} \ldots\left(\lambda_{n}\left(a_{n}\right)\right)^{m}} f\left(a_{1}^{m} x_{1}, \ldots, a_{i}^{m+1} x_{i}, \ldots, a_{n}^{m} x_{n}\right)-\right. \\
& \left.\frac{\lambda_{i}\left(a_{i}\right)}{\left(\lambda_{1}\left(a_{1}\right)\right)^{m} \ldots\left(\lambda_{n}\left(a_{n}\right)\right)^{m}} f\left(a_{1}^{m} x_{1}, \ldots, a_{n}^{m} x_{n}\right)\right)(u) \geq \\
& \lim _{m \rightarrow \infty} \omega\left(\frac{1}{\left|\left(\lambda_{1}\left(a_{1}\right)\right)^{m} \ldots\left(\lambda_{n}\left(a_{n}\right)\right)^{m}\right|} \varphi_{i}\left(a_{1}^{m} x_{1}, \ldots, a_{n}^{m} x_{n}\right)\right)(u) .
\end{aligned}
$$

By (3.2) and (3.11), we conclude that $F$ satisfies system(1.1). 
Suppose that there exists another mapping $F^{\prime}: X^{n} \rightarrow X$ which satisfies system(1.1) and (3.4). So we have

$$
\begin{aligned}
& \nu\left(F\left(x_{1}, \ldots, x_{n}\right)-F^{\prime}\left(x_{1}, \ldots, x_{n}\right)\right)(u) \\
= & \nu\left(\frac{1}{\left(\lambda_{1}\left(a_{1}\right)\right)^{m} \ldots\left(\lambda_{n}\left(a_{n}\right)\right)^{m}} F\left(a_{1}^{m} x_{1}, \ldots, a_{n}^{m} x_{n}\right)\right. \\
& -\frac{1}{\left(\lambda_{1}\left(a_{1}\right)\right)^{m} \ldots\left(\lambda_{n}\left(a_{n}\right)\right)^{m}} f\left(a_{1}^{m} x_{1}, \ldots, a_{n}^{m} x_{n}\right) \\
& +\frac{1}{\left(\lambda_{1}\left(a_{1}\right)\right)^{m} \ldots\left(\lambda_{n}\left(a_{n}\right)\right)^{m}} f\left(a_{1}^{m} x_{1}, \ldots, a_{n}^{m} x_{n}\right) \\
& \left.-\frac{1}{\left(\lambda_{1}\left(a_{1}\right)\right)^{m} \ldots\left(\lambda_{n}\left(a_{n}\right)\right)^{m}} F^{\prime}\left(a_{1}^{m} x_{1}, \ldots, a_{n}^{m} x_{n}\right)\right)(u) \\
\geq & T\left\{\Psi_{m}\left(a_{1}^{m} x_{1}, \ldots, a_{n}^{m} x_{n},\left|\left(\lambda_{1}\left(a_{1}\right)\right)^{m} \ldots\left(\lambda_{n}\left(a_{n}\right)\right)^{m}\right| u\right),\right. \\
& \left.\Psi_{m}\left(a_{1}^{m} x_{1}, \ldots, a_{n}^{m} x_{n},\left|\left(\lambda_{1}\left(a_{1}\right)\right)^{m} \ldots\left(\lambda_{n}\left(a_{n}\right)\right)^{m}\right| u\right)\right\},
\end{aligned}
$$

which tends to 1 as $m \rightarrow \infty$ by (3.3). Therefore $F=F^{\prime}$.

This completes the proof.

We conclude the following corollary if we assume $\lambda_{i}(a)=a^{i}$ in Theorem(3.1).

Corollary 3.2. Let $\varphi_{i}: X^{n} \rightarrow Z$ for $i \in\{1, \ldots, n\}$ be a mapping such that

$$
\left\{\begin{array}{l}
\tilde{\varphi}_{i}=\tilde{\varphi}_{i}\left(x_{1}, \ldots, x_{n}, u\right)=\omega\left(\frac{1}{\left|a_{1} a_{2}^{2} \ldots a_{i}^{i}\right|} \varphi_{i}\left(a_{1} x_{1}, \ldots, a_{i-1} x_{i-1}, x_{i}, \ldots, x_{n}\right)\right)(u) \\
\Phi_{1}=\Phi_{1}\left(x_{1}, \ldots, x_{n}, u\right)=\tilde{\varphi}_{1}\left(x_{1}, \ldots, x_{n}, u\right) \\
\Phi_{i}=\Phi_{i}\left(x_{1}, \ldots, x_{n}, u\right)=T\left(\tilde{\varphi}_{i}\left(x_{1}, \ldots, x_{n}, u\right), \Phi_{i-1}\left(x_{1}, \ldots, x_{n}, u\right)\right) \\
\lim _{m \rightarrow \infty} \Phi_{n}\left(a_{1}^{m} x_{1}, \ldots, a_{n}^{m} x_{n},\left|a_{1}^{m} a_{2}^{2 m} \ldots a_{n}^{n m}\right| u\right)=1
\end{array}\right.
$$

and

$$
\lim _{m \rightarrow \infty} \omega\left(\frac{1}{\left|a_{1}^{m} a_{2}^{2 m} \ldots a_{n}^{n m}\right|} \varphi_{i}\left(a_{1}^{m} x_{1}, \ldots, a_{n}^{m} x_{n}\right)\right)(u)=1
$$

and

$$
\left\{\begin{array}{l}
\Phi_{m}^{*}=\Phi_{m}^{*}\left(x_{1}, \ldots, x_{n}, u\right)=\Phi_{n}\left(a_{1}^{m} x_{1}, \ldots, a_{n}^{m} x_{n},\left|a_{1}^{m} a_{2}^{2 m} \ldots a_{n}^{n m}\right| u\right) \\
\Psi_{0}=\Phi_{0}^{*}\left(x_{1}, \ldots, x_{n}, u\right)=\Phi_{n}\left(x_{1}, \ldots, x_{n}, u\right) \\
\Psi_{m}=\Psi_{m}\left(x_{1}, \ldots, x_{n}, u\right)=T\left(\Phi_{m}^{*}\left(x_{1}, \ldots, x_{n}, u\right), \Psi_{m-1}\left(x_{1}, \ldots, x_{n}, u\right)\right) \\
\Psi=\Psi\left(x_{1}, \ldots, x_{n}, u\right)=\lim _{m \rightarrow \infty} \Psi_{m}=1 .
\end{array}\right.
$$

for all $u>0, x_{i} \in X$ and $a_{i} \in \mathbb{K} \backslash\{0\}, i=1, \ldots, n$. Let $f: X^{n} \rightarrow Y$ be a mapping 
satisfying

$$
\left\{\begin{array}{l}
\nu\left(f\left(a_{1} x_{1}, x_{2}, \ldots, x_{n}\right)-a_{1} f\left(x_{1}, \ldots, x_{n}\right)\right)(u) \geq \omega\left(\varphi_{1}\left(x_{1}, \ldots, x_{n}\right)\right)(u) \\
\nu\left(f\left(x_{1}, a_{2} x_{2}, \ldots, x_{n}\right)-a_{2}^{2} f\left(x_{1}, \ldots, x_{n}\right)\right)(u) \geq \omega\left(\varphi_{2}\left(x_{1}, \ldots, x_{n}\right)\right)(u) \\
\quad \vdots \\
\nu\left(f\left(x_{1}, \ldots, a_{i} x_{i}, \ldots, x_{n}\right)-a_{i}^{i} f\left(x_{1}, \ldots, x_{n}\right)\right)(u) \geq \omega\left(\varphi_{i}\left(x_{1}, \ldots, x_{n}\right)\right)(u) ; \\
\quad \vdots \\
\nu\left(f\left(x_{1}, \ldots, x_{n-1}, a_{n} x_{n}\right)-a_{n}^{n} f\left(x_{1}, \ldots, x_{n}\right)\right)(u) \geq \omega\left(\varphi_{n}\left(x_{1}, \ldots, x_{n}\right)\right)(u)
\end{array}\right.
$$

for all $u>0, x_{i} \in X$ and $a_{i} \in \mathbb{K} \backslash\{0\}, i=1, \ldots, n$. Then there exists a unique mapping $F: X^{n} \rightarrow Y$ satisfying system(1.1) for $\lambda_{i}\left(a_{i}\right)=a_{i}^{i}$ and

$$
\nu\left(f\left(x_{1}, \ldots, x_{n}\right)-F\left(x_{1}, \ldots, x_{n}\right)\right)(u) \geq \Psi
$$

for all $u>0$ and $x_{i} \in X, i=1, \ldots, n$.

\section{References}

[1] C. Alsina, B. Schweizer and A. Sklar, On the definition of a probabilistic normed space, Aequationes Math., 46(1993), 91-98.

[2] T. Aoki, On the stability of the linear transformation in Banach spaces, J. Math. Soc. Japan, 2(1950), 64-66.

[3] L. M. Arriola and W. A. Beyer, Stability of the Cauchy functional equation over p-adic fields, Real Anal. Exchange, 31(2005/2006), 125-132.

[4] C. Baak and M. S. Moslehian, On the Stability of Orthogonally Cubic Functional Equations , Kyungpook Math. J., 47(2007), 69-76.

[5] D. Deses, On the representation of non-Archimedean objects, Topology and its Applications, 153(2005), 774-785.

[6] M. Eshaghi Gordji, M. B. Ghaemi and H. Majani, Generalized Hyers-Ulam-Rassias Theorem in Menger Probabilistic Normed Spaces, Discrete Dynamics in Nature and Society, Volume 2010, Article ID 162371, 11 pages.

[7] M. Eshaghi Gordji, M. B. Ghaemi, H. Majani and C. Park, Generalized Ulam-Hyers Stability of Jensen Functional Equation in Š erstnev PN Spaces, Journal of Inequalities and Applications, Volume 2010, Article ID 868193, 14 pages.

[8] M. Eshaghi Gordji, H. Khodaei, Solution and stability of generalized mixed type cubic, quadratic and additive functional equation in quasi-Banach spaces, Nonlinear Anal., 71(2009), 5629-5643. 
[9] M. Eshaghi Gordji and H. Khodaei, Stability of Functional Equations, LAP LAMBERT Academic Publishing, 2010.

[10] M. Eshaghi Gordji and M. B. Savadkouhi, Stability of a mixed type cubic-quartic functional equation in non-Archimedean spaces, Appl. Math. Lett.' 23(10)(2010), 1198-1202.

[11] P. Găvruta, A generalization of the Hyers-Ulam-Rassias stability of approximately additive mappings, J. Math. Anal. Appl., 184(1994), 431-436.

[12] M. B. Ghaemi, H. Majani and M. Eshaghi Gordji, Approximately Quintic And Sextic Mappings On The Probabilistic Normed Spaces, Bull. Korean Math. Soc., 49(2)(2012), 339-352.

[13] O. Hadzić, A fixed point theorem in Menger spaces, Publ. Inst. Math. (Beograd), T20(1979), 107-112.

[14] O. Hadzić, Fixed point theorems for multivalued mappings in probabilistic metric spaces, Fuzzy Sets Syst., 88(1997), 219-226.

[15] D. H. Hyers, On the stability of the linear functional equation, Proc. Natl. Acad. Sci., 27(1941), 222-224.

[16] K.-W. Jun and Y.-H. Lee, A Generalization of the Hyers-Ulam-Rassias Stability of the Pexiderized Quadratic Equations, II, Kyungpook Math. J., 47(2007), 91-103.

[17] Y.-S. Jung and K.-H. Park, On the Generalized Hyers-Ulam-Rassias Stability for a Functional Equation of Two Types in p-Banach Spaces, Kyungpook Math. J., 48(2008), 45-61.

[18] A. K. Katsaras and A. Beoyiannis, Tensor products of non-Archimedean weighted spaces of continuous functions, Georgian Mathematical Journal, 6(1999), 33-44.

[19] A. Khrennikov, non-Archimedean Analysis: Quantum Paradoxes, Dynamical Systems and Biological Models, Kluwer Academic Publishers, Dordrecht, 1997.

[20] D. Mihet, The stability of the additive Cauchy functional equationin non-Archimedean fuzzy normed spaces, Fuzzy Sets Syst., 161(2010), 2206-2212.

[21] A. K. Mirmostafaee, Hyers-Ulam Stability of Cubic Mappings in non-Archimedean Normed Spaces, Kyungpook Math. J., 50(2010), 315-327.

[22] A. K. Mirmostafaee, M. S. Moslehian, Stability of additive mappings in nonArchimedean fuzzy normed spaces, Fuzzy Sets Syst., 160(2009), 1643-1652.

[23] L. Narici, E. Beckenstein, Strange terrain-non-Archimedean spaces, Amer. Math. Mon., 88(9)(1981) 667-676.

[24] P. J. Nyikos, On some non-Archimedean spaces of Alexandrof and Urysohn, Topology and its Applications, 91(1991), 1-23.

[25] C. Park, D. H. Boo and Th. M. Rassias, Approximately addtive mappings over $p$-adic fields, J. Chungcheong Math. Soc., 21(2008), 1-14.

[26] C. Park, M. Eshaghi Gordji, M. B. Ghaemi and H. Majani, Fixed points and approximately octic mappings in non-Archimedean 2-normed spaces, J. Ineq. Appl., 2012, 2012: 289 doi:10.1186/1029-242X-2012-289.

[27] K.-H. Park and Y.-S. Jung, On the Generalized Hyers-Ulam-Rassias Stability of Higher Ring Derivations, Kyungpook Math. J., 49(2009), 67-79. 
[28] Th. M. Rassias, On the stability of the linear mapping in Banach spaces, Proc. Amer. Math. Soc., 72(1978), 297-300.

[29] B. Schweizer and A. Sklar, Probabilistic Metric Spaces, North-Holland, NewYork, 1983.

[30] A. N. Šerstnev, On the motion of a random normed space, Dokl. Akad. Nauk SSSR, 149(1963), 280-283, English translation in Soviet Math. Dokl., 4(1963), 388-390.

[31] S. M. Ulam, Problems in Modern Mathematics, Chapter VI, Science Editions, Wiley, New York, 1964.

[32] V. S. Vladimirov, I. V. Volovich and E. I. Zelenov, $p$-adic Analysis and Mathematical Physics, World Scientific, 1994. 